\title{
Conduction at domain walls in oxide multiferroics
}

\author{
J. Seidel ${ }^{1,2 \star \dagger}$, L. W. Martin ${ }^{2,3 \star}$, Q. He ${ }^{1}$, Q. Zhan ${ }^{2}$, Y.-H. Chu ${ }^{2,3,4}$, A. Rother ${ }^{5}$, M. E. Hawkridge ${ }^{2}$, \\ P. Maksymovych ${ }^{6}$, P. Yu' ${ }^{1}$, M. Gajek ${ }^{1}$, N. Balke', S. V. Kalinin ${ }^{6}$, S. Gemming ${ }^{7}$, F. Wang ${ }^{1}$, G. Catalan ${ }^{8}$, \\ J. F. Scott ${ }^{8}$, N. A. Spaldin ${ }^{9}$, J. Orenstein ${ }^{1,2}$ and R. Ramesh ${ }^{1,2,3}$
}

\begin{abstract}
Domain walls may play an important role in future electronic devices, given their small size as well as the fact that their location can be controlled. Here, we report the observation of room-temperature electronic conductivity at ferroelectric domain walls in the insulating multiferroic $\mathrm{BiFeO}_{3}$. The origin and nature of the observed conductivity are probed using a combination of conductive atomic force microscopy, high-resolution transmission electron microscopy and first-principles density functional computations. Our analyses indicate that the conductivity correlates with structurally driven changes in both the electrostatic potential and the local electronic structure, which shows a decrease in the bandgap at the domain wall. Additionally, we demonstrate the potential for device applications of such conducting nanoscale features.
\end{abstract}

C orrelated oxide systems are an exciting and challenging area of condensed-matter research, with their interacting and competing charge, spin, orbital and lattice degrees of freedom forming new electronic and magnetic phases ${ }^{1,2}$. These phases can be controlled through stress, optical excitation and electric and magnetic fields and have great potential for applications in the fields of spintronics, information storage and communications. Among the correlated oxides, the multiferroics, which show more than one type of ferroic order in the same phase, are attracting particular interest ${ }^{3-6}$. The defining characteristic of a ferroic material is an order parameter (electric polarization in ferroelectrics, magnetization in ferromagnets or spontaneous strain in ferroelastics) that has different, energetically equivalent orientations, the orientation of which can be selected using an applied field. This often leads to the appearance of domains of differently oriented regions, separated by domain walls, coexisting in a sample ${ }^{7}$. Such domain walls will become more technologically important as the dimensions of individual elements in devices continue to shrink.

Although the morphology and properties of domains and their walls have been studied for more than 50 years, in recent times there has been increasing focus on novel functionality at domain walls $s^{8-12}$. For example, it has been predicted theoretically that the ferroelectric walls in magnetoelectric multiferroics can be ferromagnetic even if the domains themselves are antiferromagnetic ${ }^{9-11}$. Conversely, spin rotation across ferromagnetic domain walls in insulating ferromagnets can induce a local polarization in the walls of an otherwise non-polar material ${ }^{5,12}$. Experimentally, unusual functional properties of domain walls have also been observed: preferential doping along domain walls has been reported to induce two-dimensional superconductivity in $\mathrm{WO}_{3-x}$ (ref. 13) and enhanced resistivity in phosphate ${ }^{14}$, whereas in paraelectric, non-polar $\mathrm{SrTiO}_{3}$ the ferroelastic domain walls seem to be ferroelectrically polarized ${ }^{15}$. Local probes, particularly those based on scanning probe microscopy ${ }^{14}$, are invaluable in observing such effects at the nanoscale.

Here we describe the observation of a new behaviourroom-temperature electrical conductivity-at ferroelectric domain walls in the prototypical multiferroic $\mathrm{BiFeO}_{3}$ (BFO). BFO is a room-temperature G-type antiferromagnet $\left(T_{\mathrm{N}} \sim 650 \mathrm{~K}\right)$ and a rhombohedral ferroelectric $\left(T_{\mathrm{C}} \sim 1,103 \mathrm{~K}\right)$, with a large spontaneous ferroelectric polarization $\left(\sim 90 \mu \mathrm{C} \mathrm{cm}^{-2}\right)$ along the pseudocubic 111 directions ${ }^{16,17}$. Such rhombohedral ferroelectrics possess $71^{\circ}, 109^{\circ}$ and $180^{\circ}$ domain walls forming on $\{101\},\{100\}$ and planes that satisfy the requirement that $\pm h \pm k+l=0$, respectively ${ }^{18}$. All three wall orientations have been observed in BFO (refs 19,20).

Epitaxial BFO films $(\sim 100 \mathrm{~nm}$ thick $)$ were grown using laser molecular-beam epitaxy in (111), (110) and (100) orientations, using carefully controlled single-crystal $\mathrm{SrTiO}_{3}$ substrates. A thin $50 \mathrm{~nm}$ layer of epitaxial $\mathrm{SrRuO}_{3}$ was used as a bottom electrode for electrical-contact purposes. Ferroelectric domains were imaged using piezoresponse force microscopy (PFM) as described previously ${ }^{19}$. Controlled ferroelectric domain patterns were written using PFM by applying a d.c. voltage to the probe tip. Local electrical conductivity was measured using high-resolution conductive atomic force microscopy (c-AFM) (details of measurements are provided in the Methods section) by applying a bias voltage (below the polarization switching voltage) between the conductive AFM tip and the bottom electrode of the sample.

100 -nm-thick epitaxial films grown on (110) surfaces show a two-variant ferroelectric domain structure in the as-grown states with average domain sizes between 5 and $10 \mu \mathrm{m}$. On electrical switching at high fields, all three variations of domain walls can be created ${ }^{21}$; therefore, we begin our study on these (110)-oriented films. The typical morphology is shown in Fig. 1a. We measured the RMS roughness of the films to be $\sim 0.5 \mathrm{~nm}$ and there are no observable surface features, either before or after switching,

${ }^{1}$ Department of Physics, University of California, Berkeley, 94720 California, USA, ${ }^{2}$ Materials Sciences Division, Lawrence Berkeley National Laboratory, 94720 California, USA, ${ }^{3}$ Department of Materials Science and Engineering, University of California, Berkeley, 94720 California, USA, ${ }^{4}$ Department of Materials Science and Engineering, National Chiao Tung University, HsinChu, 30010, Taiwan, ${ }^{5}$ Institute of Structure Physics, Triebenberg Laboratory, Technische Universität Dresden, DE-01062, Germany, ${ }^{6}$ Center for Nanophase Materials Science, Oak Ridge National Laboratory, 37831 Tennessee, USA, ${ }^{7}$ Forschungszentrum Dresden-Rossendorf, 01314 Dresden, Germany, ${ }^{8}$ Department of Earth Sciences, University of Cambridge, Cambridge CB2 3EQ, UK,

${ }^{9}$ Materials Department, University of California, Santa Barbara, 93106 California, USA. ${ }^{\star}$ These authors contributed equally to this work.

†e-mail: jseidel@berkeley.edu. 

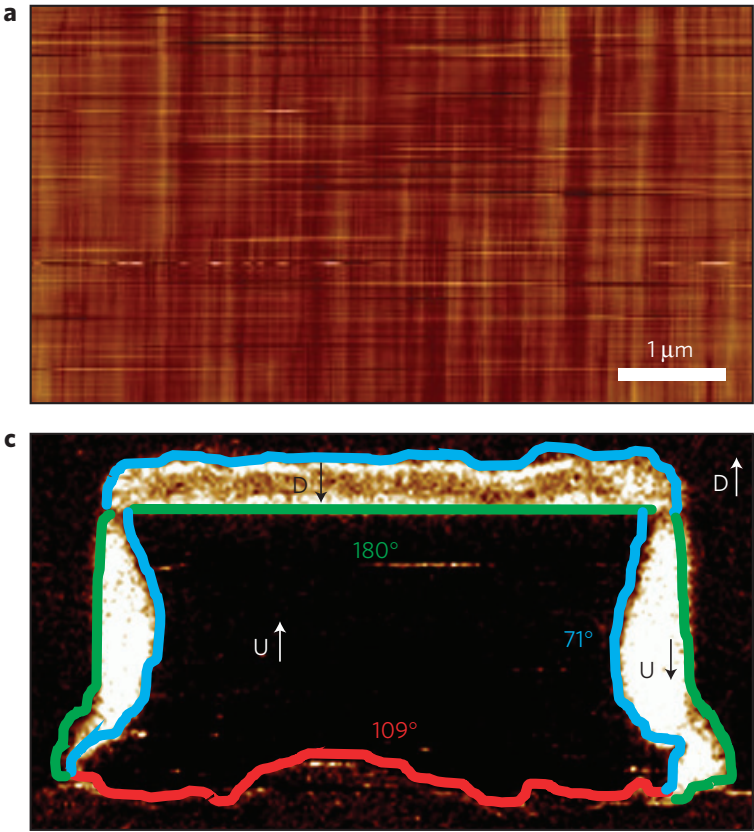

b
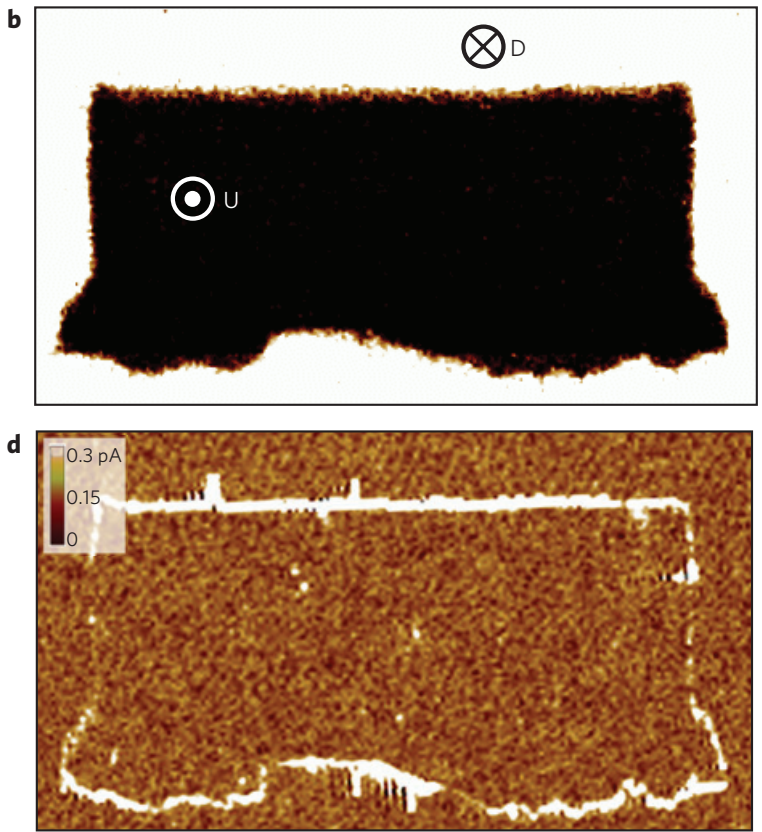

Figure 1 | Conductivity at different wall types. a, Topography of a BFO(110) thin film, r.m.s. roughness of $0.5 \mathrm{~nm}$. b, Out-of-plane PFM image of a written domain pattern in a monodomain BFO(110) film showing the out-of-plane polarization component of the domains to be either down, labelled as ' $\mathrm{D}$ ' (white), or up, labelled as 'U' (black). c, In-plane PFM image of a written domain pattern in a monodomain BFO(110) film showing all three types of domain wall, that is, $71^{\circ}$ (blue), $109^{\circ}$ (red) and $180^{\circ}$ (green), as inferred from the combination of both out-of-plane and in-plane PFM images. In these images, both the out-of-plane ( $U$ or D) component and the in-plane projection of the polarization direction (shown as an arrow) are also labelled. $\mathbf{d}$, Corresponding c-AFM image showing conduction at both $109^{\circ}$ and $180^{\circ}$ domain walls; note the absence of conduction at the $71^{\circ}$ domain walls.
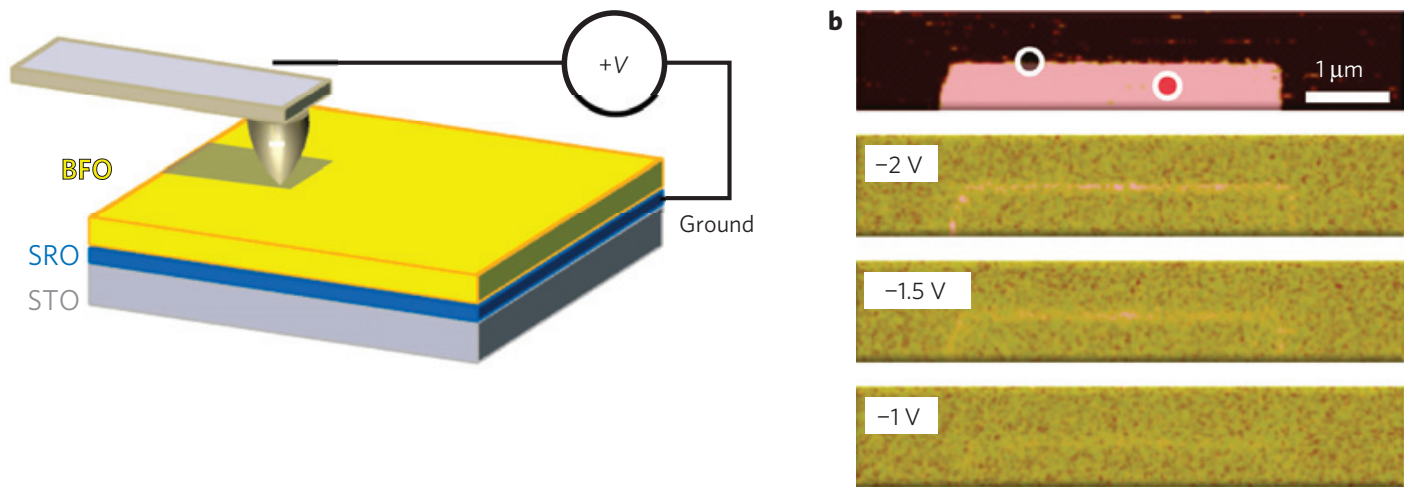

c

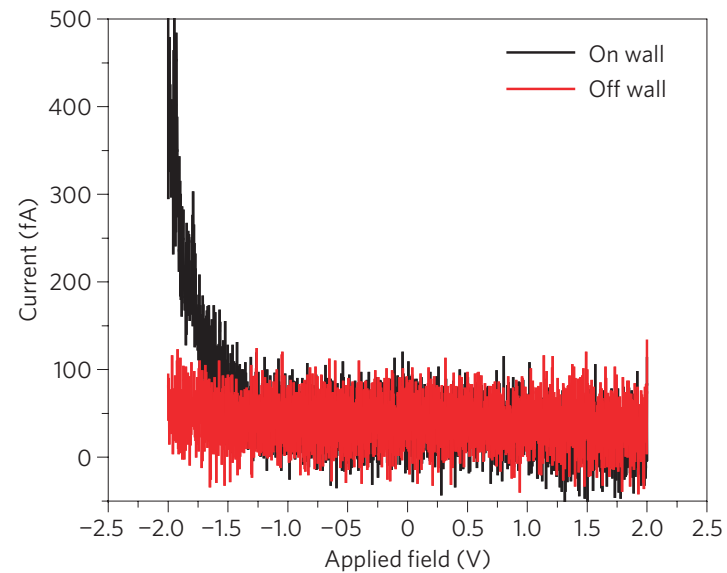

d

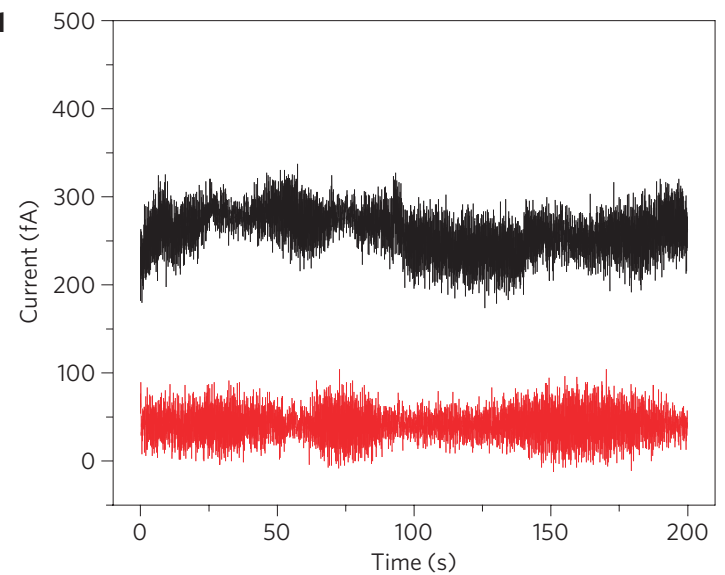

Figure 2 | Conduction at domain walls. a, Schematic illustration of the experimental c-AFM set-up. b, (Top) Out-of plane PFM image of a written $180^{\circ}$ domain in a monodomain BFO(110) sample and (lower) corresponding c-AFM current maps for $-1,-1.5$ and $-2 \mathrm{~V}$ sample bias made with a Pt-coated tip. c, $I-V$ curves taken both on the domain wall (black) and off the domain wall (red) reveal Schottky-like behaviour. $\mathbf{d}$, Time dependence of the current both on the wall (black) and off the wall (red) at an applied sample bias of $-2 \mathrm{~V}$. Results are qualitatively similar for $\mathrm{N}$-doped diamond tips. 

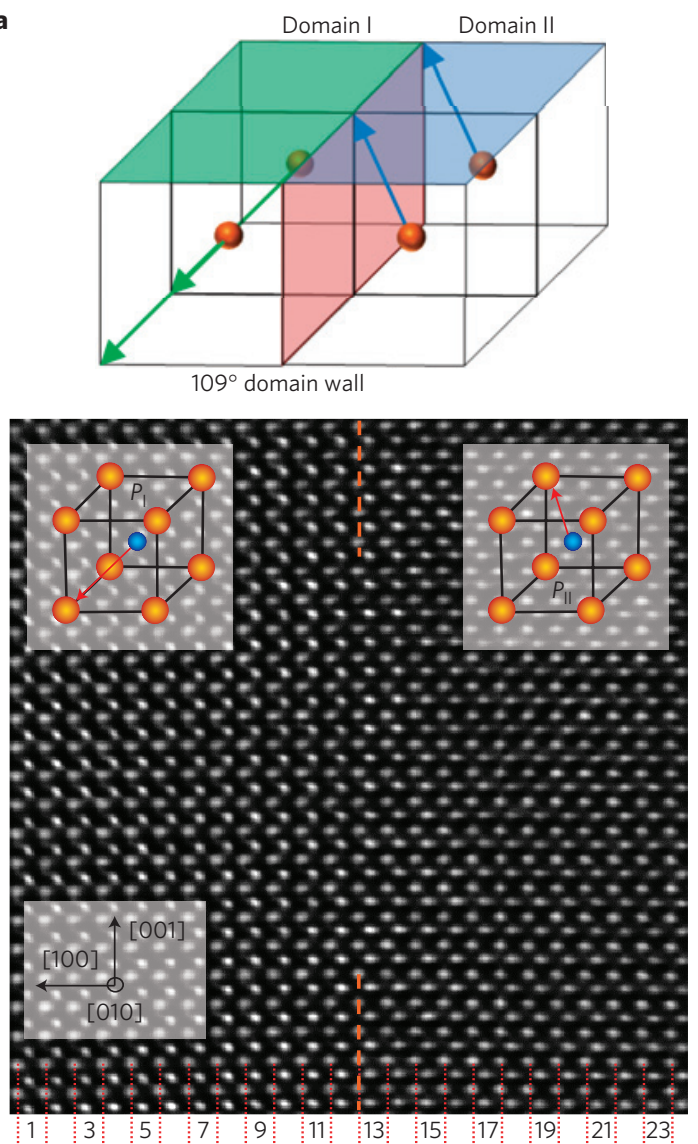

b

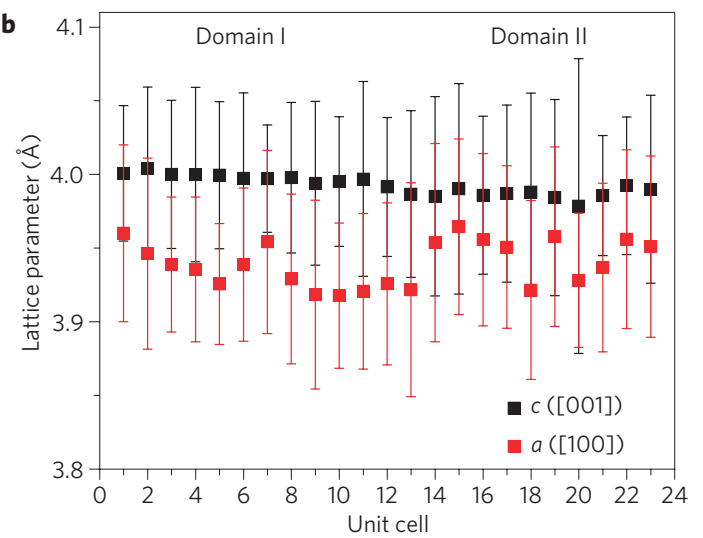

c

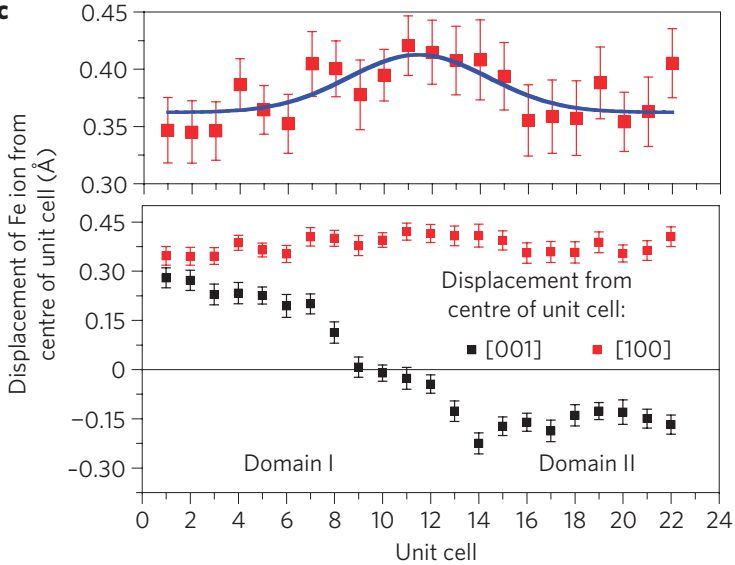

Figure 3 | Structural analysis of domain walls. a, Schematic diagram of $109^{\circ}$ domain wall and exit-wave-reconstructed $\mathrm{HRTEM}$ image of a $109^{\circ}$ domain wall imaged along the [010] zone axis. b, Extracted $a$ and $c$ lattice parameters for each unit cell across the domain wall. c, Extracted Fe-ion displacement relative to the Bi lattice for each unit cell across the domain wall. A close-up (upper panel) reveals an increase in the component of polarization perpendicular to the domain wall.

corresponding to the conducting features. In Fig. 1c, we show the in-plane PFM image of a written domain pattern controlled to have all three domain-wall types. The complicated domain shapes occur only when the high voltages required to stabilize all three domain-wall variants are applied ${ }^{21}$. The various domain-wall types are determined using both in-plane (Fig. 1c) and out-of-plane (Fig. 1b) PFM images and are labelled accordingly. Our main result is shown in the corresponding c-AFM trace (Fig. 1d): the occurrence of electrical conduction at $109^{\circ}$ and $180^{\circ}$ domain walls, and the absence of conduction at $71^{\circ}$ domain walls. BFO films grown on (001)- and (111)-oriented substrates also consistently show conduction at $109^{\circ}$ and $180^{\circ}$ domain walls (Supplementary Information, Fig. S1); in no cases do $71^{\circ}$ domain walls show conduction within the resolution of our measurements.

Next we discuss in detail our c-AFM measurements on the (110)-oriented BFO films. A schematic diagram of the experimental set-up is shown in Fig. 2a. The spatial resolution of the technique is limited by the tip radius of $\sim 20 \mathrm{~nm}$. Figure $2 \mathrm{~b}$ (top panel) shows a PFM image of two domains separated by a $180^{\circ}$ domain wall. The corresponding c-AFM images (lower panels) show enhanced conduction at the domain wall for applied bias voltages of -1 to $-2 \mathrm{~V}$. Current-voltage $(I-V)$ curves (Fig. 2c) show resistive behaviour within the domain (red data) and Schottky-like behaviour suggesting activated conduction at the domain wall (black data). We repeated our $I-V$ measurements with a number of different c-AFM tip materials_-including $\mathrm{Pt}$ and $\mathrm{N}$-doped diamond-and found similar Schottky-like behaviour with slightly shifted conduction onsets. Furthermore, the current is persistent

\section{Table 1 | Electronic structure at ferroelectric domain walls.}

\begin{tabular}{lll}
$\begin{array}{l}\text { Domain } \\
\text { wall type }\left({ }^{\circ}\right)\end{array}$ & $\begin{array}{l}\text { Electrostatic } \\
\text { potential step } \\
(\mathrm{eV})\end{array}$ & $\begin{array}{l}\text { Change in } \\
\text { bandgap }(\mathrm{eV})\end{array}$ \\
\hline 71 & 0.02 & 0.05 \\
109 & 0.15 & 0.10 \\
180 & 0.18 & 0.20 \\
\hline
\end{tabular}

Calculated values of the potential step and reduction in bandgap at all three domain-wall types.

over a timescale of at least $3 \mathrm{~min}$, which is limited by the drift in our scanning system (Fig. 2d). These time-dependent data indicate that the origin of this current is not displacement of domain walls. Additional details of conduction at domain walls are provided in the Supplementary Information (Supplementary Information, Fig. S2). Ultrahigh-vacuum-based c-AFM measurements were used to further probe the nature of conduction and $I-V$ characteristics of the conducting domain walls-including the observation of enhanced current values.

To understand the observed electrical conductivity, we performed a combined transmission electron microscopy (TEM) and density functional theory (DFT) study of the domain-wall structure and properties. The structure of domain walls in ferroelectric perovskites and related materials has been the focus of several recent DFT (refs 18,22) and TEM (refs 23-25) studies, and the structural distortions associated with ferroelectric domain 
walls are now reasonably well characterized. However, studies of the associated electronic properties are in their infancy ${ }^{26}$. We chose the $109^{\circ}$ domain wall (shown schematically in Fig. 3a) for our study because we do not obtain conduction at $71^{\circ}$ domain walls and because imaging of $180^{\circ}$ domain walls with high-resolution TEM (HRTEM) presents practical problems in terms of locating the wall. (For completeness, HRTEM images of $71^{\circ}$ domain walls are shown in Supplementary Information, Fig. S3.) We use (001)-oriented samples for the TEM analysis, because we can easily control the density of $109^{\circ}$ domain walls during growth for this orientation. TEM images were acquired using the exit-wave reconstruction approach to eliminate the effects of objective-lens spherical aberrations; such images can be directly interpreted in terms of the projection of the atomic columns ${ }^{27}$. A representative image for such a $109^{\circ}$ domain wall taken along the [010] zone axis of a cross-sectioned $\mathrm{BFO} / \mathrm{SRO} / \mathrm{STO}(001)$ film, where SRO is $\mathrm{SrRuO}_{3}$ and $\mathrm{STO}$ is $\mathrm{SrTiO}_{3}$, is shown in Fig. 3a. Analysis of this image enables us to determine the lattice parameter in the plane of the film $(a)([100])$ and the lattice parameter out of the plane of the film (c) ([001]) (Fig. 3b). As expected, the in-plane lattice parameter is slightly smaller and the out-of-plane lattice parameter larger than the values in bulk BFO (3.96 А; ref. 16) owing to the strain inherent in the epitaxial films. In addition, we find that both the in-plane and out-of-plane film lattice parameters are unchanged in the vicinity of the domain wall. We also extract the relative displacement of the $\mathrm{Fe}$ ion with respect to the Bi sublattice, and resolve it into components parallel $([001])$ and perpendicular ([100]) to the domain wall (which lies in a (100) plane running vertically across the image and labelled with a dashed orange line) by quantitative analysis of the HRTEM data (Fig. 3c); this distance is representative of the local polarization. The component of the displacement parallel to the domain wall (along [001]) decreases in magnitude to zero at the centre of the domain wall before changing to the same magnitude (but opposite sign) on the other side of the wall, reflecting the change in polarization orientation of the domain. Interestingly, the perpendicular displacement component (along [100]) shows a small increase at the domain wall; we return to the implications of this later. Similar studies were completed on a $71^{\circ}$ domain wall as well (Supplementary Information, Fig. S2). Again, only minor variation in lattice parameters was observed across the domain wall. In this case a similar step in Fe-ion displacement is observed parallel to the domain wall, but we are unable to resolve a step in the perpendicular component across the wall.

To investigate the influence of these structural changes on the electronic properties, we next performed a density functional study of the structure and electronic properties for all three ferroelectric domain-wall variants (details in the Methods section). First we performed full structural optimizations of the ionic positions with the lattice parameters fixed to their experimental bulk values; in particular, the oxygen polyhedral rotations around the polar axis, which are known to have a profound effect on both the magnetic ${ }^{28}$ and electronic ${ }^{29}$ properties and cannot be easily extracted from the HRTEM data, were accurately calculated. As the sense of the oxygen rotations around the polar axis is independent of the direction of polarization along the axis ${ }^{28}$ we studied two scenarios: first we initialized the sense of rotation to be continuous across the domain boundary and second we changed the rotation sense when the polarization direction changed. As expected, we found that domain walls with continuous oxygen rotations are considerably lower in energy, because this avoids formation of an antiphase boundary associated with the octahedral rotations. In addition, we investigated domain-wall configurations centred at both the $\mathrm{Bi}-\mathrm{O}$ and $\mathrm{Fe}-\mathrm{O}$ planes and found the $\mathrm{Bi}-\mathrm{O}$ walls to be slightly lower in energy, confirming the findings of the HRTEM analysis. Our lowest-energy calculated configuration for the $109^{\circ}$ domain wall has a domain-wall energy of $206 \mathrm{~mJ} \mathrm{~m}^{-2}$.

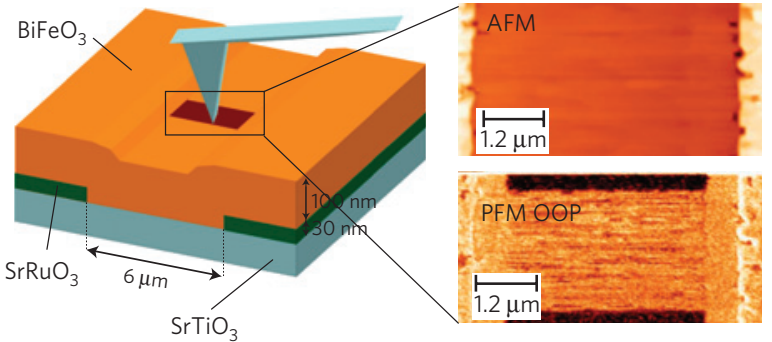

b

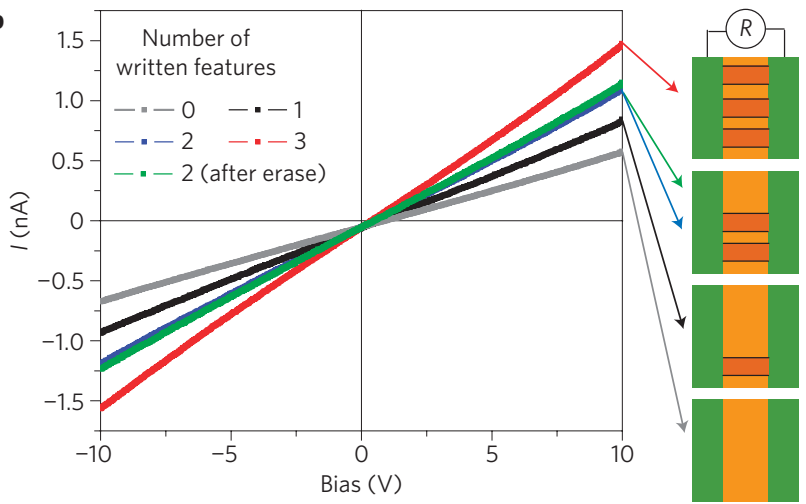

Figure 4 | Proof of concept for device application. a, Schematic illustration (left) of in-plane electrode structure (green) and how scanning probe tips can be used to controllably create conductive domain-wall features between electrodes. The images on the right show AFM (top) and out-of-plane PFM (bottom) contrast for this written domain area on a $\mathrm{BFO}(110)$ sample. $\mathbf{b}, I-V$ characteristics of the devices measured between the two in-plane electrodes show that the current can be incrementally controlled through creating or erasing the conducting domain walls.

To confirm that our calculated structure is consistent with our TEM data, we analysed the layer-by-layer polarization, defined as the sum over the bulk Born effective charges ${ }^{29}$ multiplied by the displacements of the ions from their centrosymmetric reference positions in each layer. As expected, the local polarization in the middle of the domain is close to the value calculated for bulk BFO using the same computationally and experimentally determined lattice parameters $\left(\sim 93 \mu \mathrm{C} \mathrm{cm}^{-2}\right.$; refs $\left.29-32\right)$, confirming that our supercell is large enough to capture the essential physics. Consistent with our TEM analysis, we find an abrupt change in the parallel polarization component across the domain wall and a small change in the normal component at the domain wall.

Our calculations indicate that this small change in the normal component of the polarization across the $109^{\circ}$ domain wall leads to a step in the electrostatic potential (planar and macroscopically averaged) of $0.15 \mathrm{eV}$ across the domain wall (Table 1); a similar step was computed and explained previously across $90^{\circ}$ domain walls in $\mathrm{PbTiO}_{3}$ (ref. 22). Such a potential step should enhance the electrical conductivity by causing carriers in the material to accumulate at the domain wall to screen the polarization discontinuity. Our calculations for the $180^{\circ}$ domain wall also yield a variation in the normal component of the polarization, and a corresponding potential step of $0.18 \mathrm{eV}$ (Table 1). The normal component results from the polarization rotating towards successive adjacent corners of the perovskite unit cell, through a $71^{\circ}$ and than a $109^{\circ}$ change in the polarization direction before reaching the reversed polarization ${ }^{16}$. This behaviour is in striking contrast to the $180^{\circ}$ polarization reversal in tetragonal ferroelectrics ${ }^{22}$, where the polarization changes in only one direction within the wall plane and no normal component occurs. The $71^{\circ}$ wall, however, exhibits no anomaly in the perpendicular component of the ion displacement at the centre of the wall, again consistent with our 
TEM data, and therefore exhibits a negligible electrostatic potential step at the domain wall (Table 1).

Finally, we calculate the electronic properties of our structurally optimized domain walls, in particular by comparing the layer-by-layer densities of states in the domain wall and mid-domain regions. Within the central region of the domain, we find, as expected, that the local density of states resembles that of bulk BFO, and the local Kohn-Sham bandgap is equal to the value of $1.3 \mathrm{eV}$ obtained for bulk BFO with the same choice of Hubbard parameter $U$ and exchange interaction $J, 3$ and $1 \mathrm{eV}$, respectively ${ }^{29}$. (We emphasize that, although the DFT Kohn-Sham bandgaps do not correspond to experimental bandgaps, changes in DFT gaps caused by changes in bandwidth as a consequence of small changes in structure for the same DFT implementation are qualitatively meaningful.) As the domain wall is approached, we find that the changes in the structure do indeed cause changes in the band width and the positions of the band edges. This leads in the $109^{\circ}\left(180^{\circ}\right)$ case to a $0.1 \mathrm{eV}(0.2 \mathrm{eV})$ reduction in the bandgap in the domain-wall layer from the mid-domain calculated value of $1.3 \mathrm{eV}$ (Table 1). For activated conduction at room temperature, such a change in bandgap, or in band-edge offset relative to the Fermi energy of the tip, should lead to considerable changes in conductivity. Consistent with its absence of conduction, the reduction in bandgap in the $71^{\circ}$ case is smaller $(0.05 \mathrm{eV})$ (Table 1$)$. Interestingly, the magnitude of the bandgap reduction is sensitive to the details of the lattice parameters used in the calculation; if we constrain the lattice parameters to the bulk values, the changes in the bandgap are larger than those for the relaxed structures given in Table 1. This suggests that band-structure changes at domain walls might be tunable by epitaxial strain.

Our conductivity measurements, TEM analysis and DFT calculations therefore suggest two mechanisms, which probably combine to yield the observed conductivity at the $109^{\circ}$ and $180^{\circ}$ domain walls: (1) an increased carrier density as a consequence of the electrostatic potential step at the wall and (2) a decrease in the bandgap within the wall and corresponding reduction in band offset with the c-AFM tip. Both factors are the result of structural changes at the wall.

The potential of these conducting domain walls for possible device applications is illustrated in Fig. 4. By creating a simple device structure consisting of in-plane electrodes of SRO separated by a $6 \mu \mathrm{m}$ spacing (Fig. $4 \mathrm{a}$ ), we can measure the $I-V$ characteristics of BFO films and domain walls macroscopically. Additionally, the SRO provides nearly Ohmic contacts with the BFO films and thus we can gain further insight into the conduction of the walls in the gap, without any interference from the AFM tip during the measurement process. Monodomain (110)-oriented BFO films were grown on top of the SRO in-plane electrode device structures on STO(110) substrates. Conducting domain-wall features (here are shown $180^{\circ}$ domain walls, Fig. 4a, right) that connect the two in-plane electrodes were written using PFM. Again, no morphological surface features were observed that correspond to the written domain pattern. $I-V$ measurements (Fig. $4 \mathrm{~b}$ ) reveal a step-like increase in the measured current between the two in-plane electrodes on addition of a controlled number of conducting domain walls. The steps in conduction are essentially equidistant, increase proportionally to the total number of domain walls written and show completely reversible behaviour on erasing a given feature. $I-V$ curves for zero, one, two and three domain features are shown in Fig. 4b. Such material functionality has potential application in both logic and memory applications, as the wall location (and hence electronic conduction) can be precisely controlled on the nanoscale. This demonstrates a possible rewritable, multiconfiguration device set-up that uses nanoscale conductive channels (that is, conducting domain walls). Finally, on the basis of a simple sheet-resistance model, we can estimate the resistivity of a single domain wall in BFO to be of the order of $1-10 \Omega \mathrm{m}$, which is between five and six orders of magnitude lower than for bulk BFO.

In summary, our results show that ferroelectric domain walls in multiferroic BFO show unusual local electronic transport behaviour that is quite different from that in the bulk of the material or in conventional ferroelectrics. The conductivity is consistent with our observed changes in structure at the domain wall and can be activated and controlled on the scale of the domain wall width-about $2 \mathrm{~nm}$ in BFO. The present work represents a 'proof of concept' that domain walls are discrete functional entities, which may be addressed and sensed, suggesting potential utility in novel nanoelectronic applications.

\section{Methods}

Nanoscale conductivity measurements were performed on a Digital Instruments Nanoscope-IV Multimode atomic force microscope equipped with a c-AFM application module (TUNA). The investigations were performed with commercially available $\mathrm{N}$-doped diamond-coated Si tips (NT-MDT) and Ti/Pt-coated Si tips (MikroMasch). Current-amplification settings of the c-AFM equipment of 1 and $10 \mathrm{~V} \mathrm{pA}^{-1}$ at an applicable voltage range of $\pm 12 \mathrm{~V}$ were used. For a typical scan rate of $0.5-1.0 \mu \mathrm{m} \mathrm{s}^{-1}$, the noise level was of the order of $50 \mathrm{fA}$ at a bandwidth of $250 \mathrm{~Hz}$. All data were acquired under ambient conditions and at room temperature and all such c-AFM measurements were made within a few minutes after the domain wall was created by electrical switching.

Structural investigations have been carried out using a Philips CM300 field emission gun microscope with a point-to-point resolution of $1.7 \AA$ and information resolution of $0.8 \AA$. The domain structure was investigated in cross-section [010] orientation. The representative phase of the complex electron exit wave, as shown in Fig. 3a, was reconstructed from a series of 20 high-resolution images of the BFO $109^{\circ}$ domain wall using the TrueImage software package ${ }^{33,34}$. According to the weak phase-object approximation for a thin object, the heavy Bi columns projected along the [010] direction correspond to the brightest dots and the less bright ones represent $\mathrm{Fe}$ in the phase.

DFT calculations were performed using the projector augmented wave implementation of the VASP $\operatorname{code}^{35}$ with standard projector augmented wave potentials $\left(\mathrm{Bi}, 6 s^{2} 5 d^{10} 6 p^{3}\right.$; Fe, $\left.3 p^{6} 4 s^{2} 3 d^{6} ; \mathrm{O}, 2 s^{2} 2 p^{4}\right)$ and the local spin density approximation (LSDA) $+U$ method to describe the exchange-correlation potential $^{36}$. We used a cut-off energy of $550 \mathrm{eV}$ and $U / J$ values of $3 / 1 \mathrm{eV}$; these values were shown in previous work ${ }^{29}$ to give a bandgap of $1.3 \mathrm{eV}$ for bulk rhombohedral BFO and to yield a small bandgap even in the high-symmetry cubic perovskite phase. We constructed supercells containing two oppositely polarized domains and two domain walls; each domain contained six $\mathrm{Bi}_{2} \mathrm{Fe}_{2} \mathrm{O}_{6}$ units initially set to the bulk $\mathrm{BiFeO}_{3}$ structure. Note that the systems in our calculations are in all cases electrically neutral with uncharged domain walls. We used the experimental bulk lattice parameters, with the rhombohedral angle set to $60^{\circ}$ so that the supercell could accommodate the broken symmetry of the domain wall. The Brillouin zone integration was performed on a $5 \times 5 \times 1 \Gamma$-centred Monkhorst-Pack grid incorporating a Gaussian averaging, with an energy width of $0.05 \mathrm{eV}$. Ionic positions were relaxed within the local minimum of maintaining two domains and domain walls per supercell until the total energy of the supercell converged to within $0.01 \mathrm{eV}$ and the forces acting on individual ions were below $0.03 \mathrm{eV} \AA^{-1}$. The magnetism was set to the G-type antiferromagnetic ordering observed in bulk $\mathrm{BFO}$ and remained unchanged throughout the relaxation of the domain boundary. All parameters chosen for the supercell calculations were tested in calculations for single unit cells and shown to yield well-converged total energies, with atom positions and band structures in good agreement with previous work.

Received 12 May 2008; accepted 22 December 2008; published online 25 January 2009

\section{References}

1. Tsuda, N., Nasu, K., Yanase, A. \& Siratori, K. Electronic Conduction in Oxides (Springer, 2000).

2. Imada, M., Fujimori, A. \& Tokura, Y. Metal-insulator transitions. Rev. Mod. Phys. 70, 1039-1263 (1998).

3. Spaldin, N. A. \& Fiebig, M. The renaissance of magnetoelectric multiferroics. Science 309, 391-392 (2005).

4. Eerenstein, W., Mathur, N. D. \& Scott, J. F. Multiferroic and magnetoelectric materials. Nature 442, 759-765 (2006).

5. Cheong, S.-W. \& Mostovoy, M. Multiferroics: A magnetic twist for ferroelectricity. Nature Mater. 6, 13-20 (2007).

6. Ramesh, R. \& Spaldin, N. A. Multiferroics: Progress and prospects in thin films. Nature Mater. 6, 21-29 (2007).

7. Roytburd, A. Elastic domains and polydomain phases in solids. Phase Transit. 45, 1-34 (1993).

8. Thomas, L. et al. Resonant amplification of magnetic domain-wall motion by a train of current pulses. Science 315, 1553-1556 (2007). 
9. Př́vratská, J. \& Janovec, V. Pyromagnetic domain walls connecting antiferromagnetic non-ferroelastic magnetoelectric domains. Ferroelectrics 204, 321-331 (1997).

10. Přívratská, J. \& Janovec, V. Spontaneous polarization and/or magnetization in non-ferroelastic domain walls: Symmetry predictions. Ferroelectrics 222, 23-32 (1999).

11. Goltsev, V. et al. Structure and interaction of antiferromagnetic domain walls in hexagonal $\mathrm{YMnO}_{3}$. Phys. Rev. Lett. 90, 177204 (2003).

12. Mostovoy, M. Ferroelectricity in spiral magnets. Phys. Rev. Lett. 96, 067601 (2006).

13. Aird, A. \& Salje, E. K. H. Sheet superconductivity in twin walls: Experimental evidence of $\mathrm{WO}_{3-x}$. J. Phys. Condens. Matter 10, L377-L380 (1998).

14. Bartels, M. et al. Impurity-induced resistivity of ferroelastic domain walls in doped lead phosphate. J. Phys. Condens. Matter 15, 957-962 (2003).

15. Zubko, P. et al. Strain-gradient-induced polarization in $\mathrm{SrTiO}_{3}$ single crystals Phys. Rev. Lett. 99, 167601 (2007).

16. Kubel, F. \& Schmid, H. Structure of a ferroelectric and ferroelastic monodomain crystal of the perovskite $\mathrm{BiFeO}_{3}$. Acta Crystallogr. B 46, 698-702 (1990).

17. Lebeugle, D. et al. Very large spontaneous electric polarization in $\mathrm{BiFeO}_{3}$ single crystals at room temperature and its evolution under cycling fields. Appl. Phys. Lett. 91, 022907 (2007)

18. Streiffer, S. K. et al. Domain patterns in epitaxial rhombohedral ferroelectric films. I. Geometry and experiments. J. Appl. Phys. 83, 2742-2753 (1998).

19. Zavaliche, F. et al. Multiferroic $\mathrm{BiFeO}_{3}$ films: Domain structure and polarization dynamics. Phase Transit. 79, 991-1017 (2006).

20. Béa, H. et al. Influence of parasitic phases on the properties of $\mathrm{BiFeO}_{3}$ epitaxial thin films. Appl. Phys. Lett. 87, 072508 (2005).

21. Cruz, M. P. et al. Strain control of domain-wall stability in epitaxial $\mathrm{BiFeO}_{3}$ (110) films. Phys. Rev. Lett. 99, 217601 (2007).

22. Meyer, B. \& Vanderbilt, D. Ab initio study of ferroelectric domain walls in $\mathrm{PbTiO}_{3}$. Phys. Rev. B 65, 104111 (2002).

23. Stemmer, S., Streiffer, S. K., Ernst, F. \& Rühle, M. Atomistic structure of $90^{\circ}$ domain walls in ferroelectric $\mathrm{PbTiO}_{3}$ thin-films. Phil. Mag. A 71, 713-724 (1995).

24. Floquet, N. \& Valot, C. Ferroelectric domain walls in $\mathrm{BaTiO}_{3}$ : Structural walls model interpreting fingerprints in XRPD diagrams. Ferroelectrics 234, 107-122 (1999).

25. Jia, C.-L. et al. Atomic-scale study of electric dipoles near charged and uncharged domain walls in ferroelectric films. Nature Mater. 7, 57-61 (2008).

26. Wu, X. \& Vanderbilt, D. Theory of hypothetical ferroelectric superlattices incorporating head-to-head and tail-to-tail 180 degree domain walls. Phys. Rev. B 73, 020103(R) (2006).

27. Allen, L. J., McBride, W., O'Leary, N. L. \& Oxley, M. P. Exit wave reconstruction at atomic resolution. Ultramicroscopy 100, 91-104 (2004).
28. Ederer, C. \& Spaldin, N. A. Weak ferromagnetism and magnetoelectric coupling in bismuth ferrite. Phys. Rev. B 71, 060401(R) (2005).

29. Neaton, J. B. et al. First-principles study of spontaneous polarization in multiferroic $\mathrm{BiFeO}_{3}$. Phys. Rev. B 71, 014113 (2005).

30. Li, J. F. et al. Dramatically enhanced polarization in (001), (101), and (111) $\mathrm{BiFeO}_{3}$ thin films due to epitiaxial-induced transitions. Appl. Phys. Lett. 84, 5261 (2004).

31. Ravindran, P. et al. Theoretical investigation of magnetoelectric behavior in $\mathrm{BiFeO}_{3}$. Phys. Rev. B 74, 224412 (2006).

32. Lebeugle, D. et al. Electric-field induced spin flop in $\mathrm{BiFeO}_{3}$ single crystals at room temperature. Phys. Rev. Lett. 100, 227602 (2008).

33. Coene, W. M. J., Thust, A., Op de Beeck, M. \& Van Dyck, D. Maximum-likelihood method for focus-variation image reconstruction in high resolution transmission electron microscopy. Ultramicroscopy 64, 109-135 (1996).

34. Thust, A., Coene, W. M. J., Op de Beeck, M. \& Van Dyck, D. Focal-series reconstruction in HRTEM: Simulation studies on non-periodic objects. Ultramicroscopy 64, 211-230 (1996).

35. Kresse, G. \& Furthmüller, J. Efficient iterative schemes for ab initio total-energy calculations using a plane-wave basis set. Phys. Rev. B 54, 11169-11186 (1996).

36. Anisimov, V. I., Aryasetiawan, F. \& Liechtenstein, A. I. First-principles calculations of the electronic structure and spectra of strongly correlated systems: The LDA + U method. J. Phys. Condens. Matter 9, 767-808 (1997).

\section{Acknowledgements}

The work at Berkeley is supported by the Director, Office of Science, Office of Basic Energy Sciences, Materials Sciences Division of the US Department of Energy under contract No DE-AC02-05CH1123. The authors from Berkeley would like to acknowledge the support of the National Center for Electron Microscopy, Lawrence Berkeley National Laboratory. J.S. acknowledges support from the Alexander von Humboldt Foundation. Y.H.C. would also like to acknowledge the support of the National Science Council R.O.C., under contract No NSC 97-3114-M-009-001. A.R. and S.G. acknowledge support from Deutsche Forschungsgemeinschaft through FOR 520 and Deutsche Akademische Austauschdienst through GE 1202/5-1, and N.A.S. acknowledges support from NSF DMR Award No DMR-0605852 and the Miller Institute for Basic Research in Science, UC Berkeley.

\section{Additional information}

Supplementary Information accompanies this paper on www.nature.com/naturematerials Reprints and permissions information is available online at http://npg.nature.com/ reprintsandpermissions. Correspondence and requests for materials should be addressed to J.S 